\title{
A deep learning approach based on stochastic gradient descent and least absolute shrinkage and selection operator for identifying diabetic retinopathy
}

\author{
Thirumalaimuthu Thirumalaiappan Ramanathan ${ }^{1}$, Md. Jakir Hossen ${ }^{2}$, Md. Shohel Sayeed ${ }^{1}$, \\ Joseph Emerson Raja ${ }^{2}$ \\ ${ }^{1}$ Faculty of Information Science and Technology (FIST), Multimedia University (MMU), Melaka, Malaysia \\ ${ }^{2}$ Faculty of Engineering and Technology (FET), Multimedia University (MMU), Melaka, Malaysia
}

\begin{abstract}
Article Info
Article history:

Received Jul 10, 2021

Revised Nov 25, 2021

Accepted Dec 2, 2021

Keywords:

Deep neural network

Diabetic retinopathy

LASSO

Stochastic gradient descent

Weiner filter

ABSTRACT

More than eighty-five to ninety percentage of the diabetic patients are affected with diabetic retinopathy (DR) which is an eye disorder that leads to blindness. The computational techniques can support to detect the DR by using the retinal images. However, it is hard to measure the DR with the raw retinal image. This paper proposes an effective method for identification of DR from the retinal images. In this research work, initially the Weiner filter is used for preprocessing the raw retinal image. Then the preprocessed image is segmented using fuzzy c-mean technique. Then from the segmented image, the features are extracted using grey level co-occurrence matrix (GLCM). After extracting the fundus image, the feature selection is performed stochastic gradient descent, and least absolute shrinkage and selection operator (LASSO) for accurate identification during the classification process. Then the inception v3-convolutional neural network (IV3-CNN) model is used in the classification process to classify the image as DR image or non-DR image. By applying the proposed method, the classification performance of IV3-CNN model in identifying DR is studied. Using the proposed method, the DR is identified with the accuracy of about $95 \%$, and the processed retinal image is identified as mild DR.
\end{abstract}

This is an open access article under the CC BY-SA license.

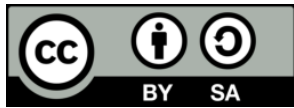

\section{Corresponding Author:}

Md. Jakir Hossen

Faculty of Engineering and Technology (FET), Multimedia University (MMU)

Melaka, Malaysia

Email: jakir.hossen@mmu.edu.my

\section{INTRODUCTION}

The artificial intelligence techniques are widely used for classification and prediction in many applications [1]-[5]. In medical diagnosis, the deep learning models are becoming more popular for identifying various diseases [6], [7]. Diabetic retinopathy (DR) is one of the serious eye diseases which needs to be accurately identified. The deficiency of insulin or incomplete activity of insulin causes diabetes mellitus which is a sugar metabolism disorder, and it is identified by increased glucose level in the blood. This increased blood glucose level damages the blood vessels through which the blood is carried to all organs of the body and results in DR. The effect of DR is increased as per the time and ends with visual blindness. The accurate and efficient functional enhancement of diabetic retinopathy can be analyzed by DR computer aided diagnosis (CAD) [8] system. Thus, the diabetic retinopathy blindness can be rectified if it is detected early and automated DR screening [9] is used for accuracy and complex tasks. To identify diabetic retinopathy issues, blood vessels from the retina should be extracted and this extraction can be performed by matched 
filter and Laplacian of Gaussian filter [10]. The pixel level analysis can be done by multiple instance learning (MIL) [11] at each level of image interpretation for accurate detection of DR lesions. For better identification of DR, the pattern classification can be performed by Micro-aneurysms [12] for classifying the changed and unchanged retinal areas.

The early detection of DR is hard, and it consumes more time for diagnosis. However, the referable DR can be diagnosed by classifying colored retinal image with the combination of CAD and deep learning algorithm [13]. The automatic detection of diabetic retinopathy from different classes of retinal images with improved features and stage classifications can be performed by convolutional neural network (CNN) [14], [15]. The convolution of irrelevant data is improved when the CNN is applied to one-dimension data set. The DR can be detected quickly by using the data from electronic health record [16] and thus the machine learning models are used with these data to obtain better accuracy. The automatic diagnosis and appropriate implication for DR is obtained by deep neural network [17] with data set of fundus DR image. The CAD is used to diagnose DR medical image data; however, the decision making is difficult and to improve the decision making of DR image, the class-enhanced attentive response discovery radiomics (CLEAR-DR) [18] is utilized. The analysis of medical image with low latency and performance of the segmentation can be improved by combining CNN with both deep learning and graphics processing unit [19]. Thus, the features and dependence of DR can be selectively achieved by cross-disease attention network [20], and this network is used only for image level observation. While detecting diabetic retinopathy medical image, it conveys the non-DR lesion as DR lesions. To compensate this medical image identification, the method based on neovessel like structures [21] can be used where the pre-existent image of retina is provided. Manually DR can be detected by analyzing the fundus retinal image which requires more time, and it is very complex to execute. However, in traditional approach the artificial intelligence [22] is utilized which play a major role in identifying DR at its early stage.

The quantitative tool that is used for identifying diabetic retinopathy is given by generating region of interest [23]; with this the images are reconstructed and then the histogram and clustering is obtained for DR image. To equalize the intensity and for improving the quality of image contrast, limited adaptive histogram equalization [24] is utilized; with this method DR image efficiency is uniform and intensity of retinal damage is improved. From the obtained uniform image, classification is performed by dividing large data set into smaller data set and to attain better accuracy level, deep CNN [25] is employed. Thus, the CNN is optimized for developing optical coherence tomography [26] and by extracting the retinal image, the performance is improved. It is necessary to classify DR image as per its severity and this severity-based classification can be performed by hyper parameter tuning inception-V4 [27], for identifying its intensity more accurately. However, diabetic eye disease known to be diabetic retinopathy is detected by evaluating the following aspects like available dataset, image processing techniques, deep learning models and then by its performance [28]. The performance and features of DR image can be extracted by using anisotropic dualtree complex wavelet transform [29]; with this, the DR medical image is obtained. The spatial representation of DR lesions can be obtained by multispectral imaging [30] in which the structure and thickness of retina is distinguished. The interior lesions of DR can be identified by employing MIL [31] which is better for irregular lesions of DR. However, the morphological variations or its complication also leads to the development of DR [32], further these techniques do not overcome diabetic risk factors and complications.

This paper proposes a method to explore suitable features for classifying DR image based on its severity level. The accuracy of DR image classification is improved by undergoing several steps where it is initiated with preprocessing technique and ends with classification process. Here, classification is performed by inception V3- CNN (IV3-CNN) to obtain better accuracy. The rest of this paper is structured as follows: the function of the entire proposed method is given in section 2 . The result and performance analysis of this proposed method is given in section 3 and our proposed method is concluded in section 4 .

\section{THE PROPOSED METHOD}

The aim of the proposed method is to detect DR. Figure 1 shows architecture of the proposed method. According to the proposed method, initially the preprocessing is performed to convert red-greenblue (RGB) image of DR into grey image and the noise present in the image is removed. This pre-processed image is segmented and then features of DR are classified, through this severity level of DR is obtained by following various classification levels. Here, IV3-CNN classifier is employed to perform the classification process. For accurate determination of DR, the input retinal image is passed through the following five steps.

Indonesian J Elec Eng \& Comp Sci, Vol. 25, No. 1, January 2022: 589-600 


\subsection{Preprocessing}

The initial stage at each image processing technique is the preprocessing and enhancement; with this the suspicious region in fundus image is detected. The removal of noise and edge reduction is performed by the preprocessing method. Here, the Weiner filter is utilized. This filter contains various advantages like edge enhancement and noise reduction. By this filter, the RGB image is converted into grey image to perform computation accurately. The Weiner filter is one of the low-pass filters and it is based on a statistical approach where the signal and noise are assumed with spectral characteristics. This filter is registered in various context for reestablishing noise degraded signal and based on the performance, the Weiner filter is said to be superior. However, the Weiner filter is also said as an adaptive filter as it evaluates mean and variance of neighboring at the situation in which the variation is maximum then the applied smoothing is minimum, likewise when the variation is low then the applied smoothing is high.

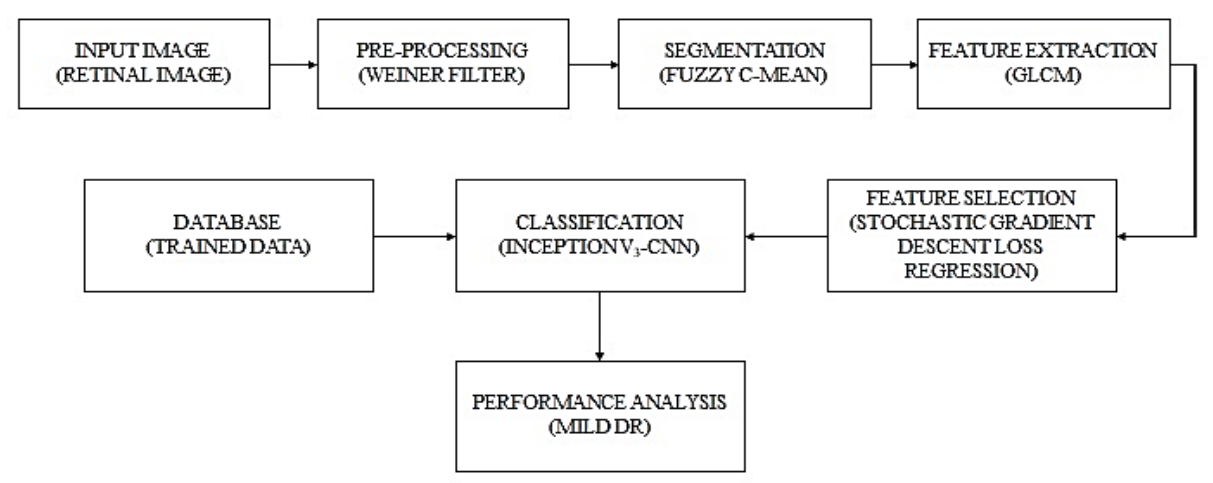

Figure 1. Architecture of the proposed method

The error that occurs in between the estimated signal and original signal is minimized by the Weiner filter. Here, the uncorrupted fundus image is represented as $\mathrm{f}$, the estimated fundus image is given as $\hat{f}$, and thus the error is measured as (1):

$$
e^{2}=E\left\{(f-\hat{f})^{2}\right\}
$$

Here, the value for argument is given by $\mathrm{E}\{$.$\} . The chance for obtaining is reduced by minimum quadratic$ error function. To accomplish this, the frequency domain is employed, and the following speculation is done; noise and image contains zero mean, and the linear functions are used to degrade the level of intensity in the estimated image. The minimum error is illustrated by following these conditions as (2):

$$
\hat{F}(u, v)=\left[\frac{H^{*}(u, v) S_{f}(u, v)}{S_{f}(u, v)|H(u, v)|^{2}+S_{f}(u, v)}\right] G(u, v)
$$

In this the estimated fundus image with frequency domain is represented as $\hat{F}(u, v)$, the transform for degradation function is given as $\mathrm{H}(\mathrm{u}, \mathrm{v})$ and the degraded image is given as $\mathrm{G}(\mathrm{u}, \mathrm{v})$, the conjugate of $\mathrm{H}(\mathrm{u}, \mathrm{v})$ is given as $\mathrm{H}^{*}(\mathrm{u}, \mathrm{v})$, and the non-degradable image's power spectrum is represented as $S_{f}(u, v)=$ $|F(u, v)|^{2}$. The product of complex value with conjugate value equates squared complex value which is the general principle of filter. Therefore,

$$
\hat{F}(u, v)=\left[\frac{1}{H(u, v)} \frac{|H(u, v)|^{2}}{|H(u, v)|^{2}+S_{n}(u, v) / S_{f}(u, v)}\right] G(u, v)
$$

here, the power spectrum of noise is given as $S_{\eta}(u, v)=|N(u, v)|^{2}$. Due to the unknowing of non-degraded fundus image, $S_{\eta}(u, v) / S_{f}(u, v)$ is substituted with constant $\mathrm{k}$. At digital image processing, the persistent power additive noise is degraded by the Weiner filter, and the parameters of the filter are area, size, and noise power. In Weiner filter, the optimal trade-off is executed among inverse filtering and noise smoothing, the noise is removed simultaneously, and the occurred blur is inverted.

\subsection{Segmentation}

The segmentation is performed in the preprocessed input image. In the segmentation method, the image is segmented into several set of regions and each region contains information like texture and 
intensity. The histogram is generated along with the segmentation to obtain normalized color intensity. The retinal vasculature segmentation is the most important as it contain detailed information about the patient's health. For the recognition of DR and to perform spatial alignment and registration of the image, an accurate segmentation is required. Here, the fuzzy c-mean is employed to perform segmentation process because it terminates false alarms. Each segmented region is considered as a fuzzy zone and other regions are considered as background. Fuzzy c-mean converts each target into a cluster where the members with same characteristics are grouped in a same class. The cluster center is evaluated by measuring the distance between cluster and pixel as (4),

$$
C_{p q}=\frac{\sum_{r=1}^{n}\left(U_{p r}\right)^{2} X_{r q}}{\sum_{r=1}^{n}\left(U_{p r}\right)^{2}}
$$

where $\mathrm{U}$ represents the membership function, $\mathrm{n}$ represents the number of pixels, $\mathrm{X}$ represents the pixel value, $\mathrm{p}$ and $\mathrm{q}$ represent the cluster amount, and $\mathrm{C}$ represents the cluster center. While measuring, the membership value becomes high when pixel is nearer to the cluster and the membership value becomes low when pixel is far from the cluster. The cluster center value and membership values are upgraded in each iteration until it satisfies the convergence criterion. In fuzzy c-mean, the pixel from one cluster has the ability to become a member of another cluster, and each pixel value is reversed for each cluster center. The presence of large number of clusters may results in downside; however, by increasing the iteration level the accuracy can be improved. Below describes the fuzzy c-mean algorithm.

In fuzzy c-mean, the group of $\mathrm{N}$ elements $\left(X=\left\{X_{1}, X_{2}, \ldots \ldots, X_{n}\right\}\right)$ are converted as a fuzzy c-mean cluster $\left(V=\left\{V_{1}, V_{2}, \ldots \ldots, V_{N}\right\}\right)$.

Step 1. Cluster center ' $C$ ' is selected randomly.

Step 2. Fuzzy membership ' $\mu_{i j}$ ' is evaluated by

$$
\mu_{i j}=\frac{1}{\sum_{k=1}^{c}\left(\frac{d_{i j}}{d_{i k}}\right)^{(2 l m-1)}}
$$

Step 3. The fuzzy center $V_{j}$ is assessed by

$$
V_{j}=\frac{\left(\sum_{i=1}^{n}\left(\mu_{i j}\right)^{m} x_{i}\right)}{\left(\sum_{i=1}^{n}\left(\mu_{i j}\right)^{m}\right)}, \forall=1,2, \ldots c
$$

Step 4. Both the step 2 and 3 are repeated till achieving the minimum ' $\mathrm{J}$ ' value else $\left\|U^{(K+1)}-U^{(K)}\right\|<B$, B $=0.01$.

$$
J=\sum_{i=1}^{M} \sum_{j=1}^{C} u_{i j}^{m}\left\|X_{i}-C_{j}\right\|^{2}
$$

Where ' $\mathrm{K}$ ' represents the iteration step, ' $\mathrm{B}$ ' represents the termination criterion in the range of $[0,1], \mathrm{U}=$ $\left(\mu_{i j}\right) N * C$, 'U' represents the fuzzy membership matrix, and ' $\mathrm{J}$ ' represents the objective function. The individual clusters are obtained by selecting the fuzzy membership matrix, and the matrix is formed by clusters. Here, the lowest mean value is considered as the first cluster, the highest mean value is considered as the last cluster, and the middle mean value is considered as the middle cluster.

\subsection{Feature extraction}

In the feature extraction method, the large set of input data measurement is converted into a minimized set of features. In this method, the selected texture and retrieving features of the segmented fundus image are extracted by grey level co-occurrence matrix (GLCM). The co-occurrence matrix is considered as an intermediate matrix because it constitutes the relation among pixels that are situated in the image at different direction and co-relation. This co-occurrence matrix is used in GLCM. Thus, it is known as a second-order statistical feature extraction. In GLCM, the number of rows and columns are similar to the grey levels.

The GLCM is known as a square matrix $(\mathrm{L} \times \mathrm{L})$ for the greyscale image I with spatial dimension $\mathrm{M} \times \mathrm{N}$ and the range of grey level is given as $T=\left[t_{i, j}\right] L \times L$. The grey value transmissions are specified by each matrix elements. The special co-ordinate $(m, n)$ of each pixel in the image embraces grey value of $\mathrm{f}(\mathrm{m}, \mathrm{n})$. The nearest adjacent pixels are calculated at the location: $(\mathrm{m}+1, \mathrm{n}),(\mathrm{m}-1, \mathrm{n}),(\mathrm{m}, \mathrm{n}+1)$, and $(\mathrm{m}, \mathrm{n}-1)$.

Indonesian J Elec Eng \& Comp Sci, Vol. 25, No. 1, January 2022: 589-600 
The grey level $f(m, n)$ is varied by its corresponding grey levels to form co-occurrence matrix: $f(m+1, n), f(m-$ $1, n), f(m, n+1)$, and $f(m, n-1)$.

The co-occurrence matrix is formed by both grey level $\mathrm{i}$ and $\mathrm{j}$, also by considering the lower transitions on vertical and horizontal as given (8).

$$
t_{i, j}=\sum_{m=1}^{N} \sum_{n=1}^{N} \delta
$$

Where

$$
\delta=\left\{\begin{array}{l}
1 \text { if }\left\{\begin{array}{l}
f(m, n)=i \text { and } f(m, n+1)=j \\
f(m, n)=i \text { and } f(m+1, n)=j
\end{array}\right. \\
0 \text { otherwise }
\end{array}\right.
$$

by normalizing the transition level at co-occurrence matrix, the grey level $\mathrm{i}$ to grey level $\mathrm{j}$ transition is obtained like (10),

$$
P_{i, j}=\frac{t_{i, j}}{\sum_{i=1}^{L} \sum_{j=1}^{L} t_{i, j}}
$$

grey level of GLCM is represented as (11),

$$
P(d, \phi)=\left(\begin{array}{ccc}
P_{0,0} & P_{0,1} & P_{0, N-1} \\
P_{1,0} & : & : \\
P_{N-1,0} & P_{N-1,1} & P_{N-1, N-1}
\end{array}\right)
$$

it is a $\mathrm{N} \times \mathrm{N}$ matrix, where:

$$
P_{\mathrm{i}, \mathrm{j}}=\frac{\text { number of pixels pairs with intensity }(\mathrm{i}, \mathrm{j})}{\text { total number of pairs considered }}
$$

Here, $P_{i, j}$ defines the number of grey level pair $(\mathrm{i}, \mathrm{j})$ that raised when pixels are separated with the distance and angle $\emptyset$ and P represent the total amount of normalized element that needs to configure GLCM.

In the image, the presence of local variations, intensity among the pixels and its neighbor are measured by (13),

$$
\text { Contrast }=\sum_{i=0}^{N-1} \sum_{j=0}^{N-1}(i=j)^{2} P_{i j}
$$

where GLCM pixel is represented by $P_{i j}$.

The closeness of the GLCM image distribution is represented as (14),

$$
\text { Homogeneity }=\sum_{i=0}^{N-1} \sum_{j=0}^{N-1} \frac{P_{i j}}{(1+|i, j|)}
$$

The grey level dependency in co-occurrence matrix is known to be correlation, and it is represented as,

$$
\text { Correlation }=\sum_{i=0}^{N-1} \sum_{j=0}^{N-1} \frac{(i-\mu)\left(j-\mu_{i}\right) P_{i}}{\sigma_{i} \sigma_{j}}
$$

where the mean, and standard deviation of pixel $\mathrm{i}$ and $\mathrm{j}$ are represented as $\mu_{i}, \mu_{j}, \sigma_{i}, \sigma_{j}$.

The smoothness and uniformity of texture in the image is obtained by the squared element summation in co-occurrence matrix, which is represented as,

$$
\text { Energy }=\sum_{i=0}^{N-1} \sum_{j=0}^{N-1} P_{i j}^{2}
$$

where these obtained features are used to extract GLCM texture features of the image and features of the segmented images are retrieved by eliminating the redundant data. The feature selection process is performed using the extracted image.

\subsection{Feature selection}

After extracting the fundus image, feature selection process is performed. The feature selection process is implemented by considering the smallest data so that the data accurate prediction of extracted image can be performed by minimizing the computation time. Here, the stochastic gradient descent method 
with least absolute shrinkage and selection operator (LASSO) regression is used to perform the feature selection process by eliminating redundant and irrelevant features of the image.

The well-organized approach to obtain the finest result for smallest data is stochastic gradient descent in which the slope is referred as gradient and the slope is moved in the downward direction to influence the lowest point. Here, the downward movement is performed until it reaches the minimum point. For stochastic gradient descent, a small set of data is trained instead of training the entire data. However, the small set of data is used for each iteration and each small data set is known as batch.

The stochastic gradient descent model is said to be a discriminative learning model because it selects batch from the observed data. The size of batch is considered as one because the batch used for iteration is one and the computation of single sample is performed for gradient cost function. The performance of stochastic gradient descent is faster because it updates the information instantly after each iteration. Thus, the time required for iteration is minimized. Below describes the stochastic gradient descent algorithm.

Step 1. Initially the loss function for each iteration is considered and represented as (17),

$$
J(\theta)=(\breve{y}-y)^{2}(x)
$$

where $\breve{y}$ represents predicted value and y represents actual value. Both $\breve{y}$ and y are selected with respect to x. Step 2. Gradient ' $\nabla$ ' of the loss function is computed in step 1.

Step 3. Initial value of the features is considered randomly to begin $\theta_{0}$.

Step 4. The gradient functions are updated with the feature values.

Step 5. Step size is computed as (18),

$$
\text { Step size }=\text { Gradient } * \text { Learning rate }
$$

Step 6. New feature value is computed as, 'New value = Old value - Step size'. This new value is updated in the opposite direction of gradient.

$$
\theta_{1}=\theta_{0}-(\text { Step size } * \nabla j(\theta))
$$

Step 7. For each iteration, the samples are rearranged, and step 3 to 5 is repeated till the gradient value becomes nearer to zero.

The robust method to perform the feature selection and regularization task is LASSO technique in which the error and over fitting is minimized. The sum of parameter scores is calculated absolutely by LASSO and the restriction is applied for each score that the obtained sum should be less than upper bound level. In some case, LASSO is punished with least square regression and L1- penalty function.

The LASSO is estimated as (20),

$$
\hat{\beta}^{\text {lasso }}=\arg _{\beta} \min \left\{\frac{1}{2} \sum_{i=1}^{N}\left(y_{i}-\beta_{0}-\sum_{j=1}^{p} x_{i j} \beta_{j}\right)^{2}+\lambda \sum_{j=1}^{p}\left|\beta_{j}\right|\right\}
$$

it can also be represented as (21):

$$
\hat{\beta}^{\text {lasso }}=\arg _{\beta} \min \sum_{i=1}^{N}\left(y_{i}-\beta_{0}-\sum_{j=1}^{p} x_{i j} \beta_{j}\right)^{2}
$$

Each co-efficient in the LASSO is transformed by constant $\lambda$ and diminished to zero, and this is known as forward looking selection method. Thus, the predicted accuracy and interpretability is improved by LASSO. If the presence of predictor groups is high, LASSO selects only one and minimize others to zero. By this minimization, variability among the estimation is reduced.

The LASSO method is not constant in some applications which is the major drawback. Thus, the feature selection process is performed more accurately and quickly by the stochastic gradient descent technique with LASSO technique where the error and over fitting is minimized. With the benefit of feature selection process, the performance of classification is enhanced.

\subsection{Classification}

The classification is performed to improve the outcome of the feature selection process. Here, the IV3-CNN model is employed for the classification process. Figure 2 shows IV3-CNN architecture. In IV3$\mathrm{CNN}$ architecture, the image categorization is performed by five convolution layer, two max-pooling layer,

Indonesian J Elec Eng \& Comp Sci, Vol. 25, No. 1, January 2022: 589-600 
11 inception modules, one average pooling layer and one fully connected layer. The small nodes which are similar to each other are made into a cluster for improving the network depth and width, and thus the computation process is reduced efficiently. The network is trained by image-net dataset [33] and ground truth table is used in which 1,000 categories are identified. The intensity of pixels in each image are given to this network so that the parameters in networks are adjusted automatically to provide an accurate prediction. The obtained performance error is identified by comparing the difference among generated network output and ground truth table. This comparison is repeated for each sample image until the network becomes more appropriate.

The small sets of retinal images are further trained by Image-Net dataset by transferring it through IV3-CNN. It is finely tuned to reduce time and the accuracy of retinal image is improved. The transfer learning is combined with IV3-CNN for classifying the retinal image. The pre-trained weights that are obtained from Image-Net dataset is initialized at convolution layer to speed up the training process and the redundancy is minimized. In IV3-CNN, the retinal images are learned by fully connected layer. However, in order to train 1,000 output categories, the fully connected layer trains the Image-Net into five output categories. The DR is identified by fixed weights that are obtained from the convolution layer and maxpooling layer, and the initialized weights are updated throughout the training process. At each training, the dataset of fundus image is passed over several features and the data are retrained until the valid dataset is achieved. By this valid data, the IV3-CNN identifies the level of DR in fundus image with reduced parameters and complexity.

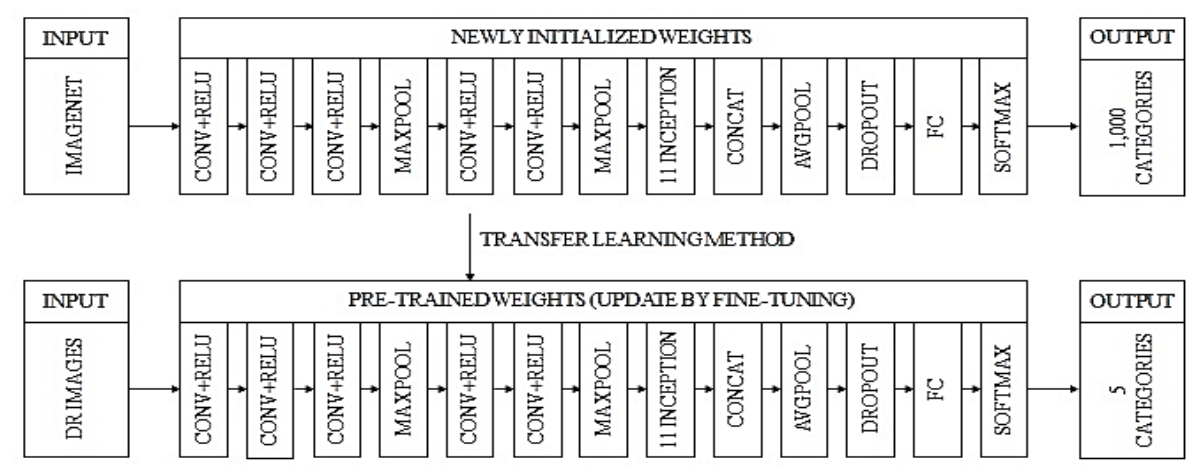

Figure 2. IV3-CNN architecture

\section{RESULTS AND DISCUSSIONS}

The proposed classification method is executed using python environment where the level of DR is traced by pairing each image with the size $256 * 256$. The severity of DR is identified by recovering several samples and the classification is performed accurately by using IV3-CNN architecture. The proposed classification method consists of several steps to achieve a high-level classification. Figure 3 shows the retina input image.

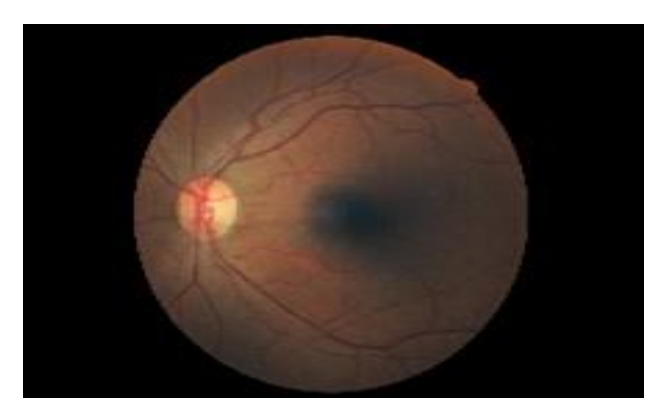

Figure 3. Input image of retina

The quality of input image is enhanced by next step. Initially the input image is in RGB and it is converted into grey image by the Weiner filter. De-noised and resized images are obtained using the Weiner filter. It allows the frequency to be passed with minimum loss by enhancing the image. Figures 4(a)-(c) show the gray scale image, noise reduced data, and filtered image, respectively. 
Threshold plays a major role in performing segmentation using which the acuteness of image is identified. The gray image is converted into binary image with a fixed threshold value where the important characteristics are pixel background and object. The threshold is performed to separate object from the background. If the pixel value is higher than the threshold value, then it appears as a bright spot and if the pixel value is less than the threshold value, then it appears as a dark spot. Figures 5(a) and (b) show the binary and enhanced images. The binary image is recovered to minimize the classification identification process.

The segmentation is performed using fuzzy c-mean method to segregate the objects as cluster. Figure 6 shows the clustered image where the DR is spotted. The retinal image is further optimized by stochastic gradient descent with LASSO regression and then the classification is performed for an accurate identification. Using the extracted features and parameters, the IV3-CNN is used to classify the image as either DR or non-DR image. The IV3-CNN classifier trained with the Image-Net dataset is tested and its performance is analyzed. Figure 7 shows the sample analysis of IV3-CNN performance where it identifies the test data as mild DR.

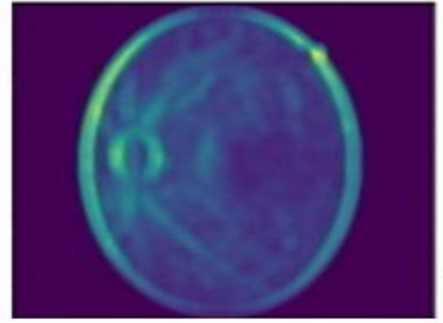

(a)

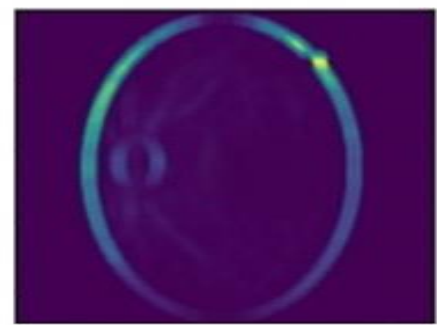

(b)

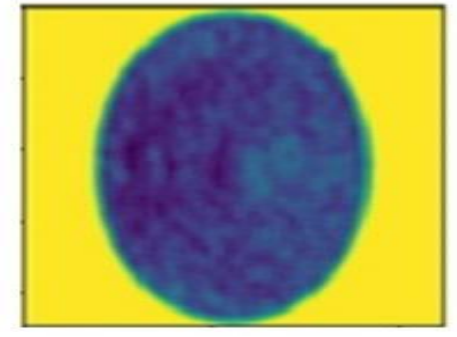

(c)

Figure 4. Filtered image of (a) gray scale image, (b) noise reduced data, (c) filtered image

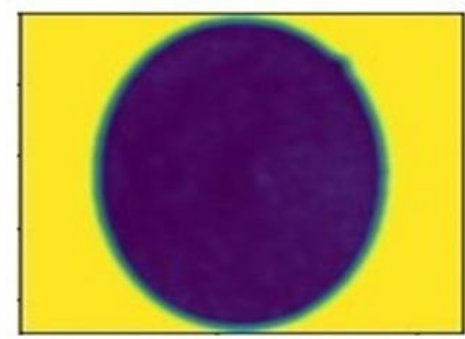

(a)

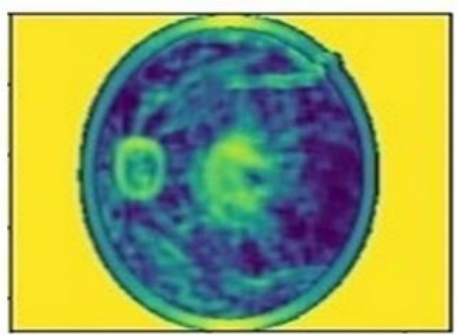

(b)

Figure 5. Enhanced image of (a) binary image and (b) enhanced image

The proposed method shows good performance in classification. The performance is analyzed using sensitivity, specificity, and classification accuracy measures which are based on the confusion matrix [34]. The sensitivity, specificity, and classification accuracy are calculated using the values of true positive (TP), true negative (TN), false positive (FP), and false negative (FN) as shown in the (22), (23), and (24).

$$
\begin{aligned}
& \text { Sensitivity }=\frac{T P}{T P+F N}=\frac{T P}{\text { All diseased people }} \\
& \text { Specificity }=\frac{T N}{T N+F P}=\frac{T N}{\text { All healthy people }} \\
& \text { Accuracy }=\frac{T P+T N}{T P+T N+F P+F N}
\end{aligned}
$$

Indonesian J Elec Eng \& Comp Sci, Vol. 25, No. 1, January 2022: 589-600 
The sensitivity identifies the TP value among the DR affected people in percentage. The specificity identifies the TN value among the healthy people in percentage. The classification accuracy identifies the rate of correct classification among the DR affected people and healthy people in percentage. Figures 8-10 show the sensitivity, specificity, and classification accuracy of the IV3-CNN classifier, respectively. Using the proposed method, the IV3-CNN classifier showed the sensitivity, specificity, and classification accuracy of about $94.3 \%, 98 \%$, and $95 \%$, respectively.

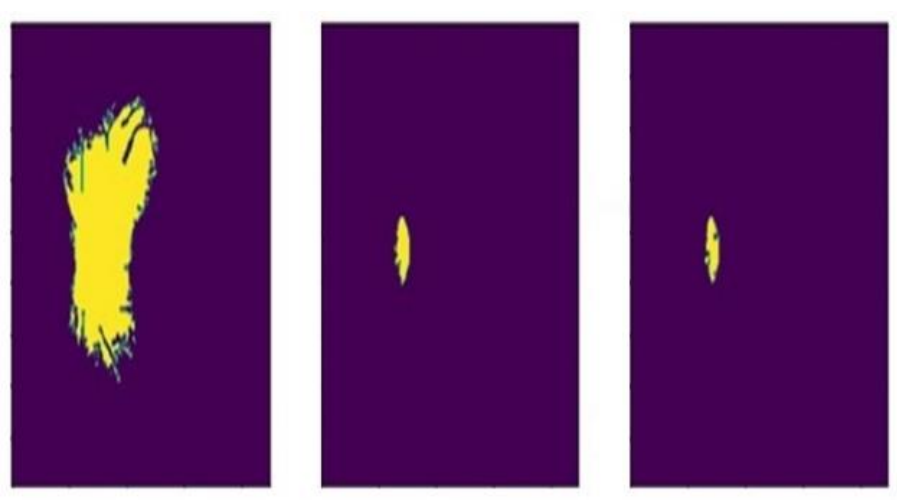

Figure 6. Clustered image

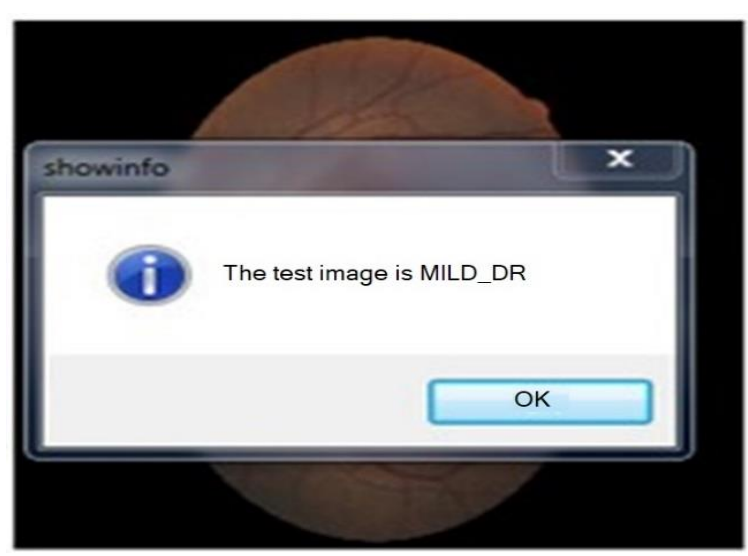

Figure 7. Analysis of DR

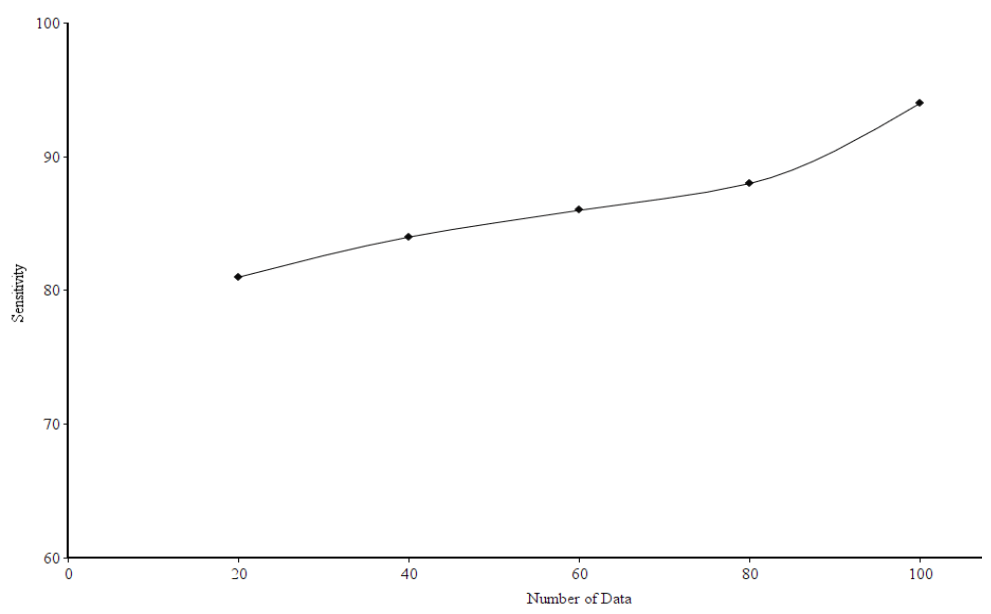

Figure 8. Sensitivity of the proposed method 


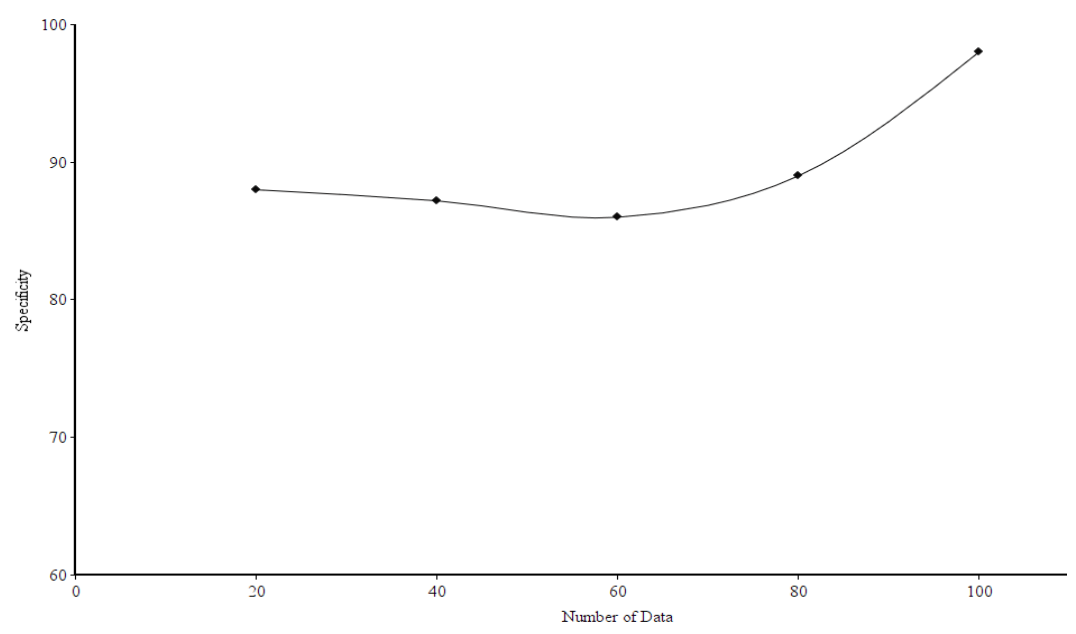

Figure 9. Specificity of the proposed method

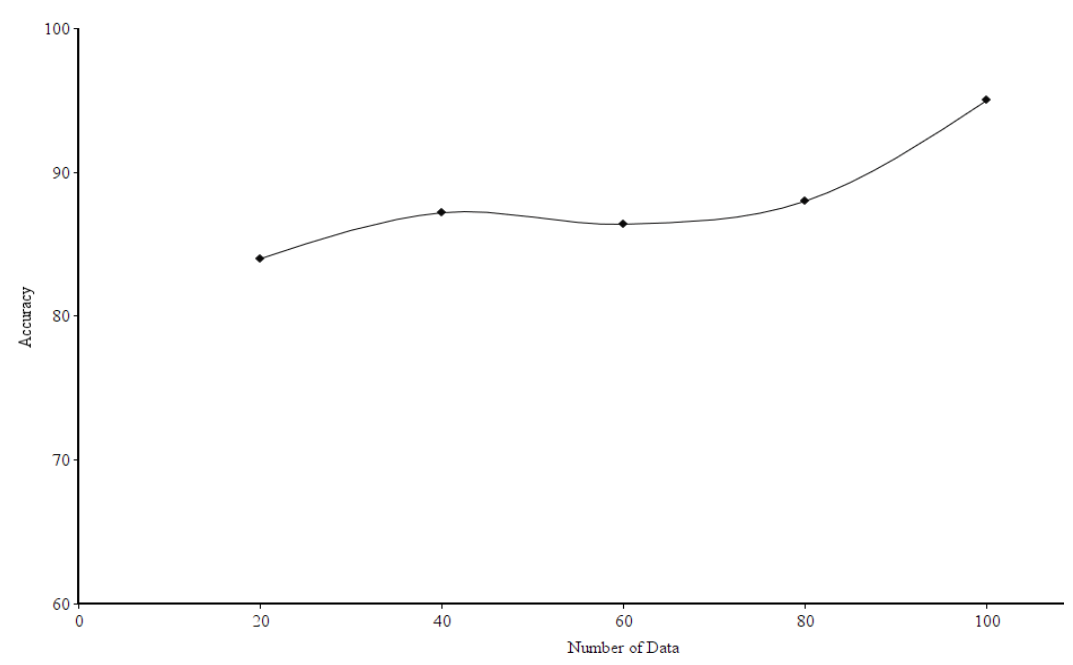

Figure 10. Classification accuracy of the proposed method

\section{CONCLUSION}

As DR is a serious eye disease caused by diabetes mellitus, there had been many research works done in artificial intelligence in the recent years to support the detection of DR from retinal images. The proposed image classification method in this paper will be added as a beneficial to the medical society in supporting the detection of DR more efficiently. The DR and its stages are identified with good accuracy using our proposed method. The input retinal image is passed through several steps where the features of retinal image are extracted, and finally the image is processed by a trained classifier. In the proposed method, the Weiner filter, fuzzy c-mean, GLCM, stochastic gradient descent, LASSO, and IV3-CNN are used for preprocessing, segmentation, feature extraction, feature selection, and classification, respectively. The classification accuracy of IV3-CNN classifier showed about $95 \%$ which proved that the proposed method is effective in identifying DR.

\section{REFERENCES}

[1] S. Aich, S. Chakraborty, and K. Hee-Cheol, "Convolutional Neural Network-Based Model for Web-Based Text Classification," International Journal of Electrical and Computer Engineering, vol. 9, no. 6, pp. 5185-5191, 2019, doi: 10.11591/ijece.v9i6.pp5185-5191. 
[2] Z. Kadim, M. A. Zulkifley, and N. Hamzah, "Deep-Learning Based Single Object Tracker for Night Surveillance," International Journal of Electrical and Computer Engineering, vol. 10, no. 4, pp. 3576-3587, 2020, doi: 10.11591/ijece.v10i4.pp3576-3587.

[3] A. A. Mas'ud, A. Jamal, S. Adewusi, and A. Sundaram, "Rotating Blade Faults Classification of a Rotor-Disk-Blade System using Artificial Neural Network," International Journal of Power Electronics and Drive Systems, vol. 12, no. 3, pp. 1900-1911, 2021, doi: 10.11591/ijpeds.v12.i3.pp1900-1911.

[4] E. A. Mahareek, A. S. Desuky, and H. A. El-Zhni, "Simulated Annealing for SVM Parameters Optimization in Student's Performance Prediction," Bulletin of Electrical Engineering and Informatics, vol. 10, no. 3, pp. 1211-1219, 2021, doi: 10.11591/eei.v10i3.2855.

[5] A. Salam, A. El Hibaoui, and A. Saif, "A Comparison of Activation Functions in Multilayer Neural Network for Predicting the Production and Consumption of Electricity Power," International Journal of Electrical and Computer Engineering, vol. 11, no. 1, pp. 163-170, 2021, doi: 10.11591/ijece.v11i1.pp163-170.

[6] E. C. Djamal, R. I. Ramadhan, M. I. Mandasari, and D. Djajasasmita, "Identification of Post-Stroke EEG Signal using Wavelet and Convolutional Neural Networks," Bulletin of Electrical Engineering and Informatics, vol. 9, no. 5, pp. 1890-1898, 2020, doi: 10.11591/eei.v9i5.2005.

[7] S. Elyassami and A. A. Kaddour, "Implementation of an Incremental Deep Learning Model for Survival Prediction of Cardiovascular Patients," IAES International Journal of Artificial Intelligence, vol. 10, no. 1, pp. 101-109, 2021, doi: 10.11591/ijai.v10.i1.pp101-109.

[8] R. F. Mansour, "Evolutionary Computing Enriched Computer-Aided Diagnosis System for Diabetic Retinopathy: A Survey," in IEEE Reviews in Biomedical Engineering, vol. 10, pp. 334-349, 2017, doi: 10.1109/RBME.2017.2705064.

[9] R. Pires, S. Avila, H. F. Jelinek, J. Wainer, E. Valle, and A. Rocha, "Beyond Lesion-Based Diabetic Retinopathy: A Direct Approach for Referral," in IEEE Journal of Biomedical and Health Informatics, vol. 21, no. 1, pp. 193-200, Jan. 2017, doi: 10.1109/JBHI.2015.2498104.

[10] S. S. Kar and S. P. Maity, "Automatic Detection of Retinal Lesions for Screening of Diabetic Retinopathy," in IEEE Transactions on Biomedical Engineering, vol. 65, no. 3, pp. 608-618, March 2018, doi: 10.1109/TBME.2017.2707578.

[11] P. Cao, F. Ren, C. Wan, J. Yang, and O. Zaiane, "Efficient Multi-Kernel Multi-Instance Learning using Weakly Supervised and Imbalanced Data for Diabetic Retinopathy Diagnosis," Computerized Medical Imaging and Graphics, vol. 69, pp. 112-124, 2018, doi: 10.1016/j.compmedimag.2018.08.008.

[12] R. K. R. Pappuru, L. Ribeiro, C. Lobo, D. Alves, and J. Cunha-Vaz, "Microaneurysm Turnover is a Predictor of Diabetic Retinopathy Progression," British Journal of Ophthalmology, vol. 103, no. 2, pp. 222-226, 2019, doi: 10.1136/bjophthalmol2018-311887.

[13] R. F. Mansour, "Deep-Learning-Based Automatic Computer-Aided Diagnosis System for Diabetic Retinopathy," Biomedical Engineering Letters, vol. 8, no. 1, pp. 41-57, 2018, doi: 10.1007/s13534-017-0047-y.

[14] M. Heisler et al., "Ensemble Deep Learning for Diabetic Retinopathy Detection using Optical Coherence Tomography Angiography," Translational Vision Science \& Technology, vol. 9, no. 2, 2020, doi: 10.1167/tvst.9.2.20.

[15] M. Shorfuzzaman, M. S. Hossain, and A. E. Saddik, “An Explainable Deep Learning Ensemble Model for Robust Diagnosis of Diabetic Retinopathy Grading," ACM Transactions on Multimedia Computing, Communications, and Applications, vol. 17, no. 3s, pp. 1-24, 2021, doi: 10.1145/3469841.

[16] R. Wang et al., "Derivation and Validation of Essential Predictors and Risk Index for Early Detection of Diabetic Retinopathy using Electronic Health Records," Journal of Clinical Medicine, vol. 10, no. 7, 2021, doi: 10.3390/jcm10071473.

[17] W. Zhang et al., "Automated Identification and Grading System of Diabetic Retinopathy using Deep Neural Networks," Knowledge-Based Systems, vol. 175, pp. 12-25, 2019, doi: 10.1016/j.knosys.2019.03.016.

[18] D. Kumar, G. W. Taylor, and A. Wong, "Discovery Radiomics With CLEAR-DR: Interpretable Computer Aided Diagnosis of Diabetic Retinopathy," in IEEE Access, vol. 7, pp. 25891-25896, 2019, doi: 10.1109/ACCESS.2019.2893635.

[19] H. Pratt, F. Coenen, D. M. Broadbent, S. P. Harding, and Y. Zheng, "Convolutional Neural Networks for Diabetic Retinopathy," Procedia Computer Science, vol. 90, pp. 200-205, 2016, doi: 10.1016/j.procs.2016.07.014.

[20] X. Li, X. Hu, L. Yu, L. Zhu, C.-W. Fu, and P.-A. Heng, "CANet: Cross-Disease Attention Network for Joint Diabetic Retinopathy and Diabetic Macular Edema Grading," in IEEE Transactions on Medical Imaging, vol. 39, no. 5, pp. 1483-1493, May 2020, doi: 10.1109/TMI.2019.2951844.

[21] T. Araújo et al., "Data Augmentation for Improving Proliferative Diabetic Retinopathy Detection in Eye Fundus Images," in IEEE Access, vol. 8, pp. 182462-182474, 2020, doi: 10.1109/ACCESS.2020.3028960.

[22] U. Ishtiaq, S. A. Kareem, E. R. M. F. Abdullah, G. Mujtaba, R. Jahangir, and H. Y. Ghafoor, "Diabetic Retinopathy Detection through Artificial Intelligent Techniques: A Review and Open Issues," Multimedia Tools and Applications, vol. 79, no. 21, pp. 15209-15252, 2020, doi: 10.1007/s11042-018-7044-8.

[23] I. P. Okuwobi, Z. Ji, W. Fan, S. Yuan, L. Bekalo, and Q. Chen, “Automated Quantification of Hyperreflective Foci in SD-OCT With Diabetic Retinopathy," in IEEE Journal of Biomedical and Health Informatics, vol. 24, no. 4, pp. 1125-1136, April 2020, doi: 10.1109/JBHI.2019.2929842.

[24] A. M. Pour, H. Seyedarabi, S. H. A. Jahromi, and A. Javadzadeh, "Automatic Detection and Monitoring of Diabetic Retinopathy Using Efficient Convolutional Neural Networks and Contrast Limited Adaptive Histogram Equalization," in IEEE Access, vol. 8, pp. 136668-136673, 2020, doi: 10.1109/ACCESS.2020.3005044.

[25] W. Chen, B. Yang, J. Li, and J. Wang, "An Approach to Detecting Diabetic Retinopathy Based on Integrated Shallow Convolutional Neural Networks," in IEEE Access, vol. 8, pp. 178552-178562, 2020, doi: 10.1109/ACCESS.2020.3027794.

[26] M. Ghazal, S. S. Ali, A. H. Mahmoud, A. M. Shalaby, and A. El-Baz, "Accurate Detection of Non-Proliferative Diabetic Retinopathy in Optical Coherence Tomography Images Using Convolutional Neural Networks," in IEEE Access, vol. 8, pp. 34387-34397, 2020, doi: 10.1109/ACCESS.2020.2974158.

[27] M. Patil et al., "Deep Hyperparameter Transfer Learning for Diabetic Retinopathy Classification," Turkish Journal of Electrical Engineering \& Computer Sciences, vol. 29, no. SI-1, pp. 2824-2839, 2021, doi: 10.3906/elk-2105-36.

[28] M. M. Islam, H. C. Yang, T. N. Poly, W. S. Jian, and Y. C. J. Li, "Deep Learning Algorithms for Detection of Diabetic Retinopathy in Retinal Fundus Photographs: A Systematic Review and Meta-Analysis," Computer Methods and Programs in Biomedicine, vol. 191, 2020, doi: 10.1016/j.cmpb.2020.105320.

[29] S. Gayathri, A. K. Krishna, V. P. Gopi, and P. Palanisamy, "Automated Binary and Multiclass Classification of Diabetic Retinopathy Using Haralick and Multiresolution Features," in IEEE Access, vol. 8, pp. 57497-57504, 2020, doi: 10.1109/ACCESS.2020.2979753. 
[30] Y. He et al., "Segmenting Diabetic Retinopathy Lesions in Multispectral Images Using Low-Dimensional Spatial-Spectral Matrix Representation," in IEEE Journal of Biomedical and Health Informatics, vol. 24, no. 2, pp. 493-502, Feb. 2020, doi: 10.1109/JBHI.2019.2912668.

[31] L. Zhou, Y. Zhao, J. Yang, Q. Yu, and X. Xu, "Deep Multiple Instance Learning for Automatic Detection of Diabetic Retinopathy in Retinal Images," IET Image Processing, vol. 12, no. 4, pp. 563-571, 2017, doi: 10.1049/iet-ipr.2017.0636.

[32] S. Chakrabarti, M. Cukiernik, D. Hileeto, T. Evans, and S. Chen, "Role of Vasoactive Factors in the Pathogenesis of Early Changes in Diabetic Retinopathy," Diabetes/Metabolism Research and Reviews, vol. 16, no, 6, pp. 393-407, 2000, doi: 10.1002/1520-7560(0000)9999:9999<::AID-DMRR157>3.0.CO;2-G.

[33] J. Deng, W. Dong, R. Socher, L.-J. Li, K. Li, and L. Fei-Fei, "ImageNet: A large-scale hierarchical image database," 2009 IEEE Conference on Computer Vision and Pattern Recognition, 2009, pp. 248-255, doi: 10.1109/CVPR.2009.5206848.

[34] W. Zhu, N. Zeng, and N. Wang, "Sensitivity, Specificity, Accuracy, Associated Confidence Interval and ROC Analysis with Practical SAS Implementations," NESUG Proceedings: Health Care and Life Sciences, Baltimore, Maryland, vol. 19, no. 97, 2010 .

\section{BIOGRAPHIES OF AUTHORS}
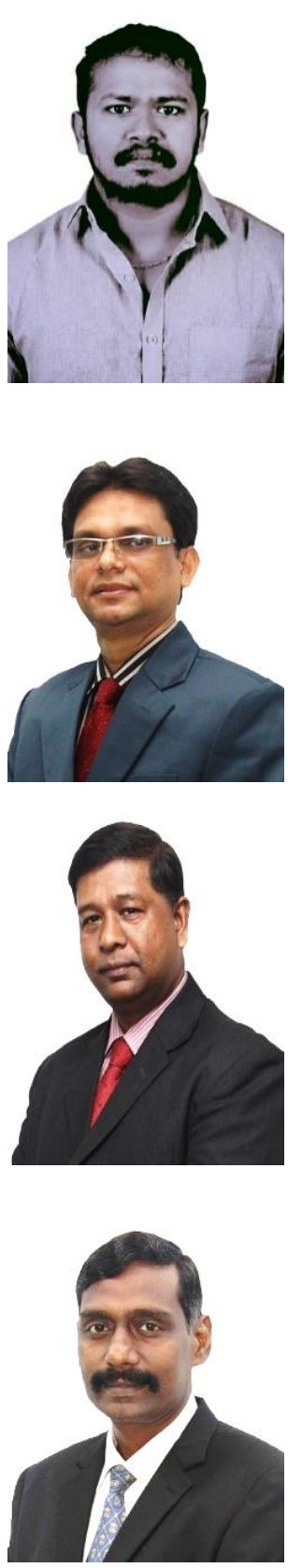

Thirumalaimuthu Thirumalaiappan Ramanathan (D) $\mathrm{g} / \mathrm{SC} \mathrm{P}$ is currently a $\mathrm{PhD}$ candidate of Faculty of Information Science and Technology, Multimedia University, Malaysia. He received the bachelor of engineering degree in computer science from Anna University, India in 2010 and the master degree in information science from University of Canberra, Australia in 2016. His research interests are application of artificial intelligence techniques in health informatics. He can be contacted at email: 1181402216@student.mmu.edu.my.

Dr. Md. Jakir Hossen (D) SC P is currently working as a Senior Lecturer in the Department of Robotics and Automation, Faculty of Engineering and Technology, Multimedia University, Malaysia. He received the master degree in communication and network engineering from Universiti Putra Malaysia (UPM), Malaysia in 2003. He received the PhD degree in smart technology and robotic engineering from Universiti Putra Malaysia (UPM), Malaysia in 2012. His research interests are artificial intelligence techniques in data analytics, robotics control, data classification and predictions. He can be contacted at email: jakir.hossen@mmu.edu.my.

Dr. Md. Shohel Sayeed (DD SC P is currently working as an Associate Professor of Faculty of Information Science and Technology, Multimedia University, Malaysia. He received the master degree in information technology from Universiti Kebangsaan Malayasia, Malaysia in 2000 and the $\mathrm{PhD}$ degree in engineering from Multimedia University, Malaysia in 2010. His research interests are biometrics, image and signal processing, cloud computing, soft computing and data mining. He can be contacted at email: shohel.sayeed@mmu.edu.my.

Dr. Joseph Emerson Raja (D) SC S P is currently working as a Senior Lecturer in the Faculty of Engineering and Technology, Multimedia University, Malaysia. He received the bachelor of engineering and master of engineering degree in computer science from University of Madras, India in 1989 and 2001 respectively. He received the PhD degree in engineering from Multimedia University, Malaysia in 2015. His research interests are application of soft computing techniques in machine condition monitoring. He can be contacted at email: emerson.raja@mmu.edu.my. 